\title{
Operational Experiment of Seamless Handover of a Mobile Router using Multiple Care-of Address Registration
}

\author{
Keiichi Shima, Yojiro Uo \\ Internet Initiative Japan Inc., Tokyo, Japan \\ Email: keiichi@iijlab.net, yuo@iijlab.net \\ Nobuo Ogashiwa \\ Intec NetCore Inc., Tokyo, Japan \\ Email: ogashiwa@inetcore.com \\ Satoshi Uda \\ Japan Advanced Institute of Science and Technology, Ishikawa, Japan \\ Email: zin@jaist.ac.jp
}

\begin{abstract}
NEMO Basic Support (NEMO BS) adds a mobility function to IPv6 routers, such a router is called as a mobile router. The network behind the mobile router (mobile network) becomes logically static. This function is considered useful when a network has a lot of nodes that do not have a mobility function but move with the network. The typical usage of this technology is the network provided in a transportation system like a bus or airplane. In the base specification of NEMO BS, a mobile router can use one IPv6 address as its attachment point at one time. Therefore, the mobile router and its mobile network will face service disruption of network connectivity while the mobile router is moving from one network to other network. In this paper, we explain two operational experiments of network mobility. One is an experiment using only the base NEMO BS function. The other is an experiment of seamless handover using multiple network interfaces to solve the problem. In these experiments, the mobile networks were actual conference networks which held a few hundred people. The result shows that the multiple interfaces usage provides one-third to one-tenth of the packet loss rate compared to the case that is not using the technology. Also most attendees of the conference did not notice the movement, which means the technology can be used for the realistic solution for the seamless handover.
\end{abstract}

Index Terms-Network Mobility, Multiple Interfaces, Seamless Handover, Real Network

\section{BACKGROUND}

Network Mobility Basic Support (NEMO BS), specified as RFC3963 [1], adds a mobility function to IPv6 routers. With the function, an entire IPv6 network served by a mobile router (a router with a mobility function) can be a mobile network. The nodes inside the mobile network can use static IPv6 addresses which never change regardless of the attachment point of the mobile router.

One of possible applications of this technology is to provide a network in moving entities, such as buses, trains, airplanes, etc. Those moving entities may connect various network access points depending on its location. The address assigned to the mobile router from the router of each access point will differ depending on each access point, since IPv6 addresses are usually assigned based on the network topology. The NEMO BS technology hides the address change of the mobile router and provides transparent access to the Internet from the nodes inside the mobile network.

Although the specification has been completed, we need more experience to deploy the technology widely. The more experience of the real operation is necessary to prove the usefulness of the technology and to find any kind of problems that may not be noticeable from the specification. Based on this policy, we performed two experiments of a mobile network that accommodated the entire conference network for the WIDE [2] 2005 autumn meeting and the WIDE 2006 spring meeting under a mobile router. The mobile router changed its point of attachment from time to time, providing transparent access to the nodes in the meeting network. The number of attendees of each meeting was almost 250 and most of them used their laptop computers to connect to the meeting network. We can think this network as a kind of moving entities which carries many people who are connected to the inside network. It can be considered as a realistic example of transportation systems, like a train.

In this paper, we will provide the basic knowledge of NEMO BS in Section II. In Section III, we briefly explain the related technologies of this experiment. In Section IV, we explain the experiment done at the WIDE 2005 autumn camp using only the base NEMO BS function. In Section V, we explain the second experiment done at the WIDE 2006 spring camp using multiple network interfaces to solve the problems that we found in the first experiment. Section VI compares the two experiments and Section VII concludes this paper. 


\section{NEMO BS OVERVIEW}

NEMO BS is a protocol which adds a mobility function to IPv6 routers. In NEMO BS, a moving router (Mobile Router, $M R$ ) has a permanent fixed address called a Home Address (HoA) and fixed network prefixes called Mobile Network Prefixes (MNPs). HoA is assigned to the MR from the network where the MR is originally attached. Such a network is called a Home Network. When the MR moves to other networks than the home network, the MR sends a message to bind its HoA and MNPs with the address assigned at the network. The message is called a Binding Update $(B U)$ message. The address at the network is called a Care-of Address (CoA) and the networks other than the home network is called Foreign Networks. The BU message is sent to a special node, called a Home Agent (HA) located in the home network. A bidirectional tunnel between the HA and the CoA of the MR is established after the binding information has been successfully exchanged. The MR may have several nodes attached to its network. Such nodes are called Mobile Network Nodes $(M N N s)$. All packets sent to the MNNs served by the MR are routed to the home network by the Internet routing mechanism. The HA intercepts the packets and forwards them to the MR using the tunnel. The MR receives the tunneled packets and forwards them again to its internal network. The packets finally reach to the MNNs. Also, the MR forwards packets sent from its MNNs using the tunnel. The nodes communicating with MNNs do not need to care about the location of the MR nor MNNs, since the communicating nodes see the MR and MNNs as if they are attached to the home network. Figure 1 illustrates the operation of Mobile IPv6.

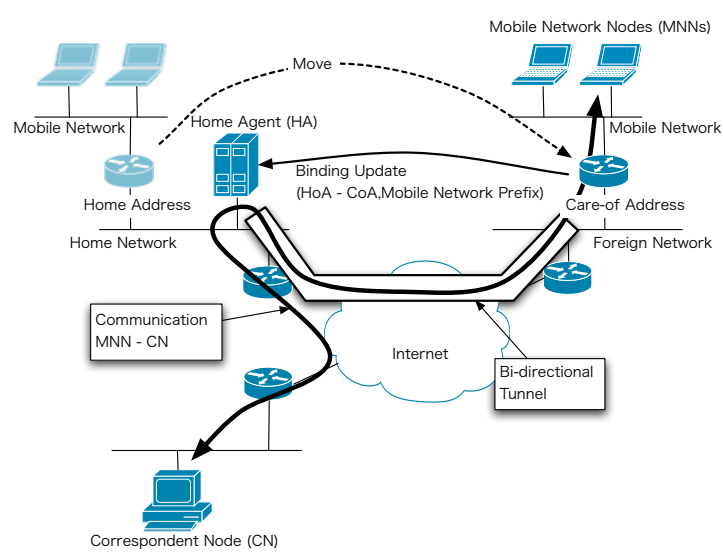

Figure 1. The concept of the NEMO technology

\section{RELATED WORKS}

In this paper, we will show our approach and the result of the experiments we made to obtain a mobile network with smooth handover. There are several approaches to make the movement seamless. One of such technologies is FMIPv6 [3].

FMIPv6 defines extensions to both a mobile node and access routers that provide foreign networks to the mobile node.
The mobile node supporting FMIPv6 will initiate movement by using some prediction mechanisms of its movement. The prediction mechanisms are not defined in the specification. Usually the mechanism is implemented by using layer 2 information. The mobile node requests a new access router information that will provide the new foreign network to where the mobile node is going to move. The mobile node notifies its movement by sending a message to the access router to which the mobile node connects currently. After receiving the message, the access router creates a bi-directional tunnel with the new access router and starts forwarding packets addressed to the mobile node. The packets tunneled from the current access router to the new access router will be buffered at the new access router until the mobile node attaches to the new foreign network. When the mobile node moves to the new foreign network, it notifies the new access router that it has completed the movement. The new access router delivers the buffered packets to the mobile node.

Even if the mobile node could not send the message that indicates the node is going to move to its current access router, the mobile node can send the message after it has moved to the new foreign network. In this case, some of the packets sent while the mobile node is moving may be lost. However, if the notification time of the movement to its old access router is smaller enough than the registration time of the mobile node to its home agent, the loss will be smaller than the case not using FMIPv6.

FMIPv6 requires changes to the infrastructure network. The access routers that provide foreign networks to the mobile node must support the protocol and must be upgraded. Also, each access router has to be authorized by each other because they creates bi-directional tunnels that forward packets of the mobile node. FMIPv6 can be used if the entire network that the mobile node attaches is managed by one operational entity (e.g. one ISP). However if we need to utilize multiple Internet service providers to get the access to the Internet, it is difficult to deploy FMIPv6.

We took another approach that utilizes multiple connections at the same time while performing handover from one foreign network to the other network. Although this mechanism requires additional equipment to the mobile node (that is, additional network interfaces), it does not require any modification to the existing infrastructure network. It requires extensions to the mobile node and its home agent. We think this approach is easy to deploy considering that the Internet is provided by the several different access service providers. The implicit assumption of this approach is the mobile node needs to be covered by at least two access networks at the same time. Otherwise, the node will lose the connectivity and will lose some packets during handover. However, considering the recent progress of wireless Internet access services, this assumption seems feasible.

\section{Moving Network Using Basic Function OnLy}

Our first experiment of NEMO BS in a real environment was the WIDE 2005 autumn camp meeting. At the meeting, a wireless network was provided to the attendees. We designed 
the network as a mobile network. In this meeting, the MR had two network interfaces, one was for external connectivity and the other was used to provide the mobile network. The mobility protocol used at this meeting was NEMO BS with no extension.

\section{A. Network Topology}

Figure 2 depicts the topology we used. The network created at the meeting site had a network 2001:200:0:ffff::/64, which was a mobile network. The MR provided the mobile network to users who participated in the meeting. The home address of the MR was 2001:200:0:fffe::4649 and the MR connected to two different networks while the meeting was being held. One network was extended from the WIDE Nara NOC (Network Operation Center), located at Nara prefecture, Japan. The network prefix was 2001:200:0:8ff::/64. The other network was extended from the WIDE K2 NOC, located at Kanagawa prefecture, Japan. The network prefix was 2001:200:0:80bb::/64. The MR acquired two different IPv6 addresses as CoAs based on its attached network. Each time the MR changed its CoA, the MR sent a message to its HA to notify that the current attachment point had been changed. The HA was placed at the WIDE Fujisawa NOC, located at Kanagawa prefecture. The address was 2001:200:0:fffe::1000. The routing information for the mobile network (2001:200:0:ffff::/64) was advertised from the HA so that all traffic to the mobile network was routed to the HA. The HA forwards all traffic addressed to the mobile network to the tunnel interface between the HA and the MR. On the other hand, all traffic generated from the IPv6 nodes inside the mobile network were forwarded by the MR to the HA using the tunnel connection.

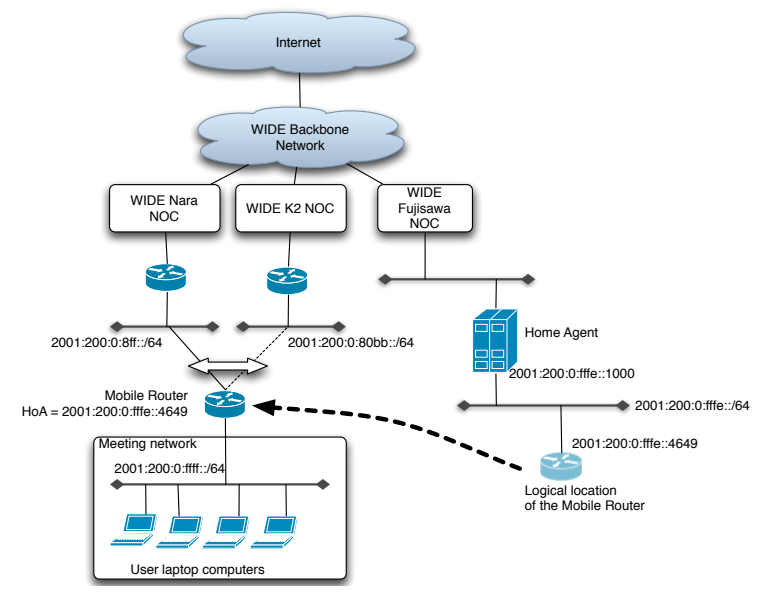

Figure 2. The network topology of the experiment at the WIDE 2005 autumn meeting

The IPv6 connections between two WIDE NOCs and access routers at the meeting site were created using IPv6 over IPv4 tunnels, because we could not get native IPv6 connection services at the meeting place.

The physical network topology is shown in Figure 3. The dotted objects mean that they are parts of IPv4 network. The
IPv4 connections to the meeting place were provided by two different ISPs, ISP-1 and ISP-2. These two ISPs and WIDE are inter-connected at one of the Internet Exchange Points in Japan. The tunnel connections, used to create two IPv6 networks, were created over the two IPv4 ISP connections respectively. We chose this configuration because we wanted to use different ISPs over those two tunnels as much as possible. If we only used one ISP service to get IPv4 access and created two IPv6 over IPv4 tunnels over the one single IPv4 ISP connection, the most part of the tunnel path would be same, since the WIDE network and ISPs in Japan usually share only one exchange point. The IPv4 path from the meeting place to the exchange point will be same even if we have two different IPv6 over IPv4 tunnels. As Figure 3 shows, we have archived almost different tunnel paths by using two different ISPs for the IPv4 connections.

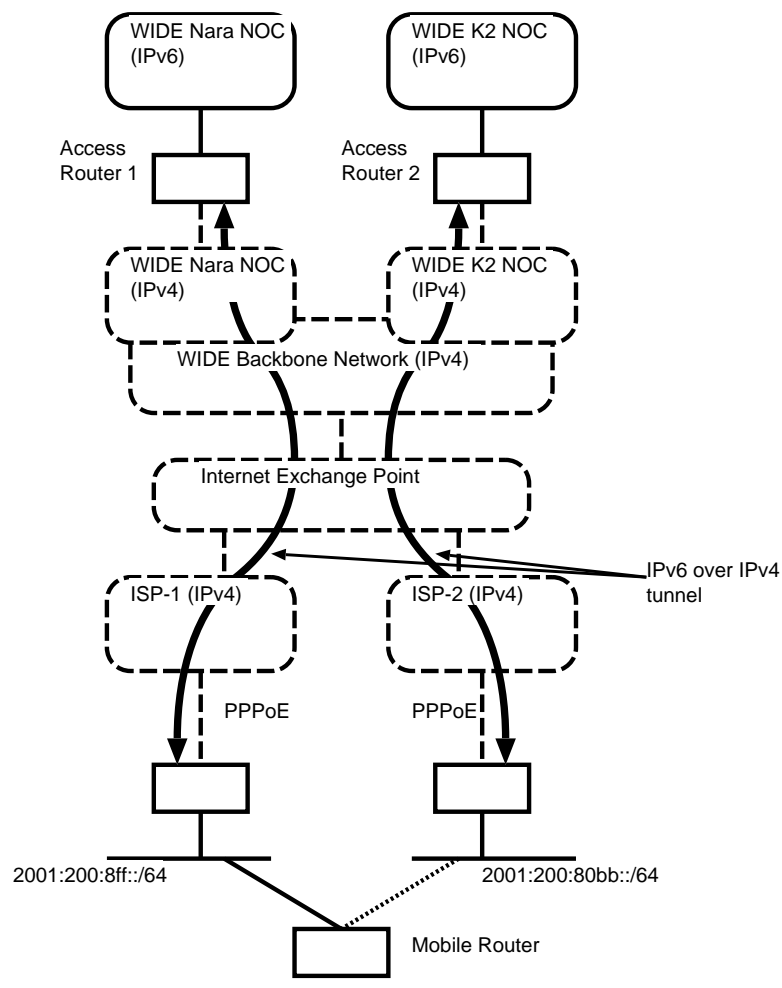

Figure 3. The physical network topology of the experiment at the WIDE 2005 autumn meeting

\section{B. System Configuration}

We used the SHISA Mobile IPv6/NEMO BS protocol stack [4] in the experiment. The hardware specification and software configuration are described in TABLE I.

The SHISA stack can be available from the KAME project [5] home page as a part of the KAME IPv6 protocol stack software. In this experiment, we used FreeBSD5.4RELEASE with the KAME snapshot as the MR and NetBSD2.0.2 with the KAME snapshot as the HA. We also used GNU Zebra [6] and ospf6d on the HA to advertise the routing information of the MR (2001:200:0:fffe::/64) and the MR's mobile network (2001:200:0:ffff::/64). 


\begin{tabular}{|c|c|c|}
\hline Node & Hardware & Software \\
\hline "MR & $\begin{array}{lll}\begin{array}{l}\text { Pentium } 4 \\
\text { memory }\end{array} & 1.8 \mathrm{G}, \quad 256 \mathrm{MB} \\
\end{array}$ & $\begin{array}{l}\text { FreeBSD5.4-RELEASE }+ \\
\text { SHISA } 20050905 \text { snapshot }\end{array}$ \\
\hline $\mathrm{HA}$ & $\begin{array}{lll}\begin{array}{l}\text { Pentium } 4 \\
\text { memory }\end{array} & 1.8 \mathrm{G}, \quad 256 \mathrm{MB}\end{array}$ & $\begin{array}{l}\text { NetBSD2.0.2 }+ \text { SHISA } \\
20050829 \text { snapshot, GNU } \\
\text { Zebra ospf6d }\end{array}$ \\
\hline
\end{tabular}

TABLE I.

SYSTEM CONFIGURATION

The MR was configured not to perform the Duplicate Address Detection (DAD) to make its movement quicker. Since the node that attached to the access networks was only the MR, we could safely omit the DAD procedure, which usually requires 1 second to complete. The MR detected its movement by receiving Router Advertisement (RA) messages from access routers. In this experiment, the access routers sent the messages every 3 seconds that is the maximum rate defined in the Neighbor Discovery specification [7].

\section{Movement Frequency}

The meeting was held for 3 days. During the meeting, we changed the movement frequency dynamically based on the user request. Figure 4 is the user interface which we used at the meeting. The movement frequency is changed every 10 minutes based on the total number of users who requested more frequent movement and less frequent movement. If the number of people who requested more frequency was greater than the number of people who requested less frequency, the movement frequency would be increased. If the comparison was opposite, the frequency would be decreased.

At the initial stage of the demonstration, we set the minimum frequency to every 20 seconds and the maximum frequency to every 60 seconds with 5 seconds as a step. However, from the day 2 of the meeting, we changed the minimum frequency to every 60 seconds and the maximum frequency to 300 seconds with 60 seconds as a step, because of the problem discussed in the next section.

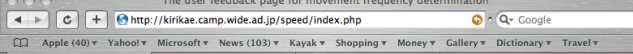 IPv6 NEMO UI}

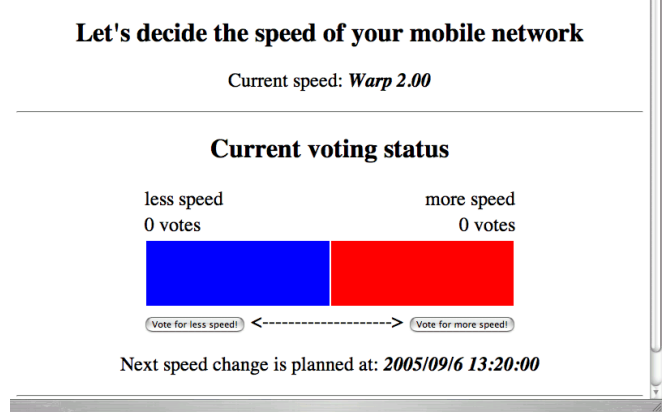

Figure 4. The user interface to vote the movement frequency used at the WIDE 2005 autumn meeting

\section{Result of the First Experiment}

Figure 5 depicts the transition of the movement frequency during the meeting. As we mentioned in the previous section, the frequency was very high in the first day of the meeting. We can see the frequency is changing dynamically and randomly, which means the participants voted their request to increase or decrease the frequency based on their feeling of the speed of the network movement. The data of the latter half of the third day is not correct because of our misconfiguration of the switch that changes the point of attachment of the MR. We manually stopped the movement for trouble shooting of the network and the movement frequency became more than 300 seconds, which could not be set by the frequency voting system.

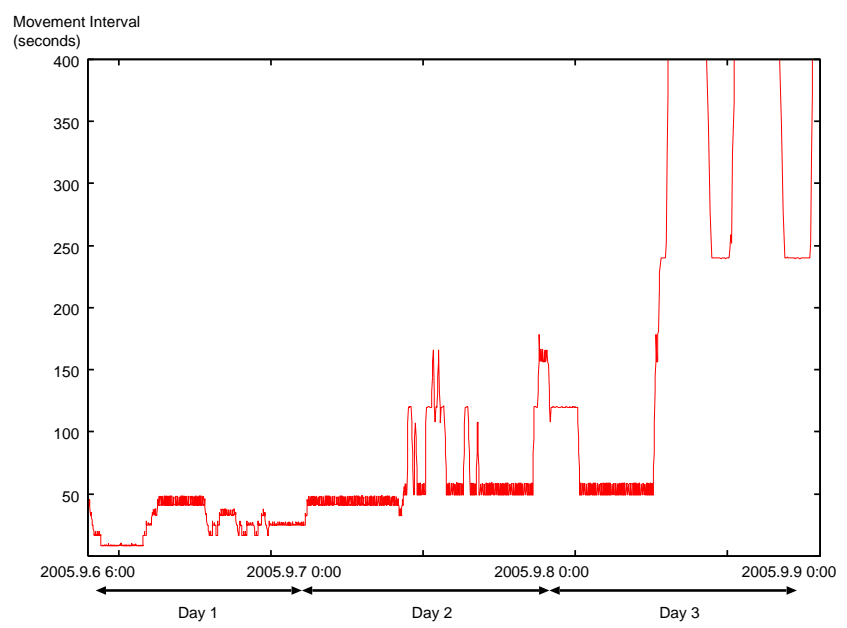

Figure 5. Transition of movement frequency at the WIDE 2005 autumn meeting

We had changed the range of the frequency that the users can change from the day 2 , since we got a lot of request that the service disruption time was too long when changing the point of attachment of the MR. In fact, there was about 20 seconds connection disruption during the MR was changing its attached network. TABLE II shows the packet loss rate using ICMPv6 echo request/reply messages from a node inside the mobile network and from the MR itself taken at the second day. The echo request messages were sent every 1 second to the destination node that was one of the IPv6 nodes located in the WIDE K2 NOC.

\begin{tabular}{l|l|l} 
From & Sent/Received & Loss rate \\
\hline \hline Node inside & $10842 / 7408$ & $31.7 \%$ \\
\hline MR & $2280 / 2249$ & $1.4 \%$
\end{tabular}

TABLE II.

PACKET LOSS RATE AT THE SECOND DAY OF THE WIDE 2005 AUTUMN MEETING

As we can see from Figure 5, the movement interval was almost 60 seconds at the second day. The mean time for the MR to detect its movement is 1.5 second, since we configured the MR not to perform the DAD procedure and configured 
the access routers to send RA messages every 3 seconds. In theory, we will lose the echo request messages during detecting movement. The theoretical packet less rate will be as follows.

\section{(DetectionTime/MovementInterval) $\times 100$}

In this case, the theoretical loss rate is $(1.5 / 60) \times 100=$ $2.5 \%$. The actual loss rate from the MR was $1.4 \%$ that was better result compared to the theoretical value. However, the packet loss rate from the internal nodes was $31.7 \%$ that was high enough to make the communication of internal nodes unstable. From Equation (1), the loss rate implies the internal node lost its connectivity almost 19 seconds on every movement. Considering that the packet loss rate was similar to the theoretical value when the echo request was sent from the MR, we thought the service disruption during the movement affected the forwarding function of the MR. That means, if we can reduce the disruption during handover, the forwarding performance will be better.

\section{Moving Network using Multiple CoAs}

We had another network mobility experiment at the WIDE 2006 spring meeting. This time, we tried to solve the problem that we had at the WIDE 2005 autumn meeting. We used the Multiple CoAs Registration mechanism [8] that makes it possible to use multiple network interfaces concurrently. At the last experiment, the MR had only one network interface. Because of this, it was impossible to avoid the service disruption during handover. If we can use multiple network interfaces concurrently, we can prepare a network interface at the new foreign network, to where the MR is going to move, before disconnecting from the old foreign network.

\section{A. Multiple CoAs Overview}

The Multiple CoAs Registration mechanism is an extension of the Mobile IPv6/NEMO BS base protocol. In the base specification of Mobile IPv6/NEMO BS, a mobile host (MH) or a mobile router can only register one $\mathrm{CoA}$ at one time. Considering the recent progress of wireless communication technology and small devices, we can expect that many devices will have multiple communication mechanisms in the future. The support for simultaneous use of multiple network interfaces are required. In the Multiple CoAs mechanism, $\mathrm{MH}$ or MR assigns unique identifiers to its network interfaces. When the $\mathrm{MH}$ or MR registers its $\mathrm{CoA}$, it also sends the unique identifier assigned to the network interface on which the CoA is assigned. The MH, MR and HA use the pair of the HoA and the unique identifier to identify the particular network interface of the MH or MR. Using this technology, the traffic between the HA and the MH or MR can be distributed over the multiple bi-directional tunnels established between them. Figure 6 shows the idea of the Multiple CoAs.

In Figure 6, the MR attaches to two foreign networks provided by the Access Router1 and the Access Router2 respectively. The MR registers its CoAs (CoA1 and CoA2) at the same time to its HA. As a result, two bi-directional tunnels are established between them. The traffic can be distributed

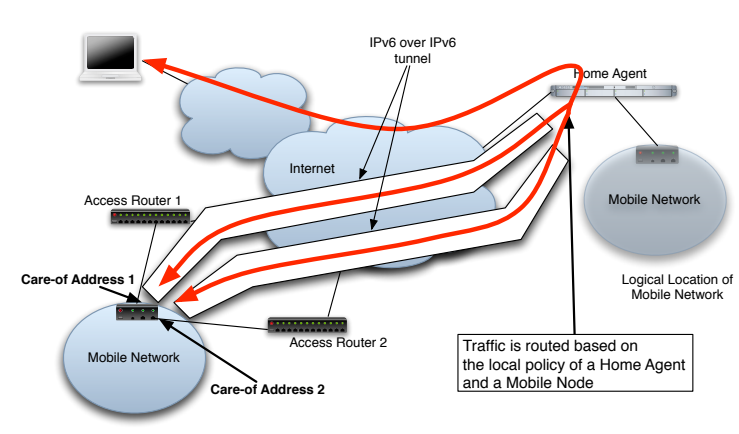

Figure 6. Multiple Care-of Address Registration and Traffic Distribution

between the two tunnels. The distribution policy is not defined in the specification and it depends on the local policy of the network operator.

\section{B. Network Topology}

Figure 7 shows the network topology used at the WIDE 2006 spring meeting. The home network was located at the WIDE K2 NOC (2001:200:0:8430::/64). The home address of the MR was 2001:200:0:8430::4649 and the address of its home agent was 2001:200:0:8430::1000. The mobile network prefix was 2001:200:0:8470::/56. This time, we allocated $/ 56$ network prefix for the meeting network, since the program committee of the meeting required more than one IPv6 subnets. Foreign networks were provided from the WIDE Fujisawa NOC. The prefixes of the foreign networks were 2001:200:0:ff60::/64, 2001:200:0:ff61::/64 and 2001:200:0:ff62::/64. Unlike the WIDE 2005 autumn meeting, we had three foreign networks at this meeting. Two of them were provided using $\mathrm{T} 1$ dedicated lines and one network was provided using a satellite link.

Similar to the WIDE 2005 autumn meeting, the IPv6 foreign networks were provided using IPv6 over IPv4 tunnels. However, unlike the previous network configuration, we did not allocate different physical paths for the two T1 lines. The reason was there was only one ISP that could provide the Internet connection to the meeting place.

\section{System Configuration}

The hardware specification and software configuration are described in TABLE III.

\begin{tabular}{l|l|lr} 
Node & Hardware & Software & \\
\hline \hline MR & Pentium4 1.8G, 256MB mem- & $\begin{array}{l}\text { NetBSD2.0.2 }+ \\
\text { 20060320 snapshot }\end{array}$ & SHISA \\
& ory & $\begin{array}{l}\text { NetBSD2.0.2 }+ \\
\text { 20060320 snapshot }\end{array}$ & SHISA \\
\hline HA & Pentium4 2.4G, 1GB memory &
\end{tabular}

TABLE III.

SYSTEM CONFIGURATION FOR THE WIDE 2006 SPRING MEETING 


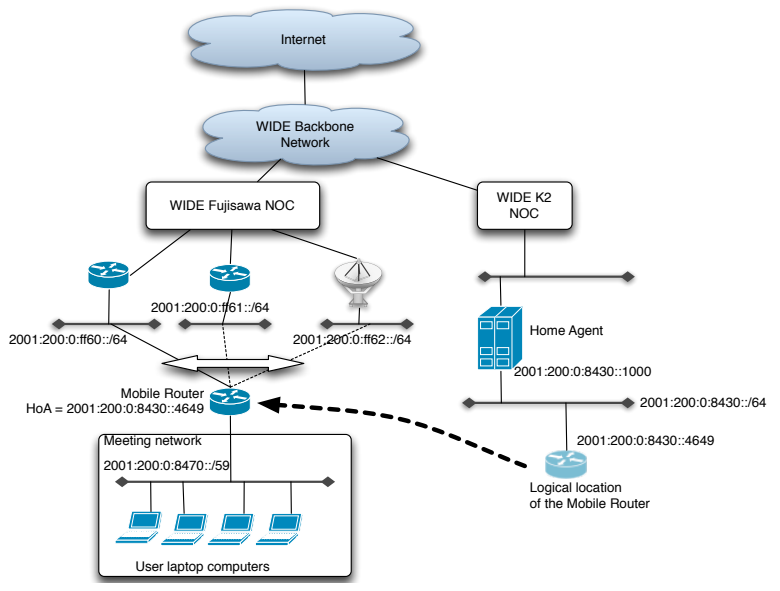

Figure 7. The network topology of the experiment at the WIDE 2006 spring meeting

In the second experiment, we used NetBSD2.0.2 with the KAME snapshot for both the MR and the HA. The routing information for the meeting network (2001:200:0:8470::/59) was distributed by route6d running on the HA.

Out traffic distribution policy was to use the network attached to the MR most recently. Although we had three different networks at this meeting, we did not use them as a method for load-balancing. We focused on the smooth handover.

The MR was configured to perform the DAD procedure. This time, the DAD procedure should not make any big effect on movement detection because the MR could complete the movement procedure before disconnecting from the old network. The interval of RA messages sent from access routers was 3 seconds.

\section{Movement Frequency}

The meeting was held for 3 days. Different from the previous meeting, the movement frequency was fixed to every 60 seconds. This time, to utilize the multiple network interfaces equipped to the MR, we configured the connection at one network interface to last 90 seconds. Figure 8 shows the strategy of the movement. The upper graph shows the strategy when the MR uses two network interfaces and the lower graph shows the case that there are three network interfaces.
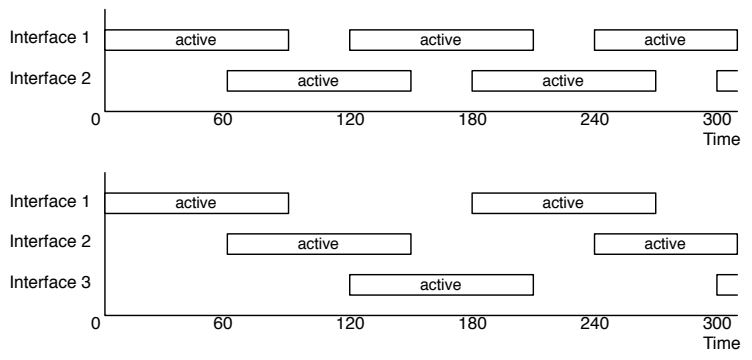

Figure 8. Movement Strategy at the WIDE 2006 spring meeting
The last 30 seconds of each connection was used as a migration period to the next connection. During this period, the MR sent a BU message to its HA. After the MR and the HA completed the registration procedure, all traffic to the meeting network and from the meeting network were sent over the new bi-directional tunnel established using the newly attached link.

\section{E. Result of the Second Experiment}

We took ICMPv6 echo request/reply statistics from MNN located at the meeting network to the node located at the WIDE K2 NOC. TABLE IV shows the result.

\begin{tabular}{l|l|l} 
& Sent/Received & Loss rate \\
\hline \hline 1st time & $20472 / 19808$ & $3.2 \%$ \\
\hline 2nd time & $39601 / 35811$ & $9.6 \%$
\end{tabular}

TABLE IV.

PACKET LOSS RATE AT THE WIDE 2006 SPRING MEETING

We also took the data transmission status using TCP and made the transition graphs of sequence number of the TCP stream. Figure 9 shows the transition of the sequence number when the MR was not moving. The $\mathrm{X}$-axis indicated the elapsed time from the beginning of the TCP stream in seconds and the Y-axis shows the relative sequence number of the TCP stream. When the MR was not moving, the sequence number drew a clear graph.

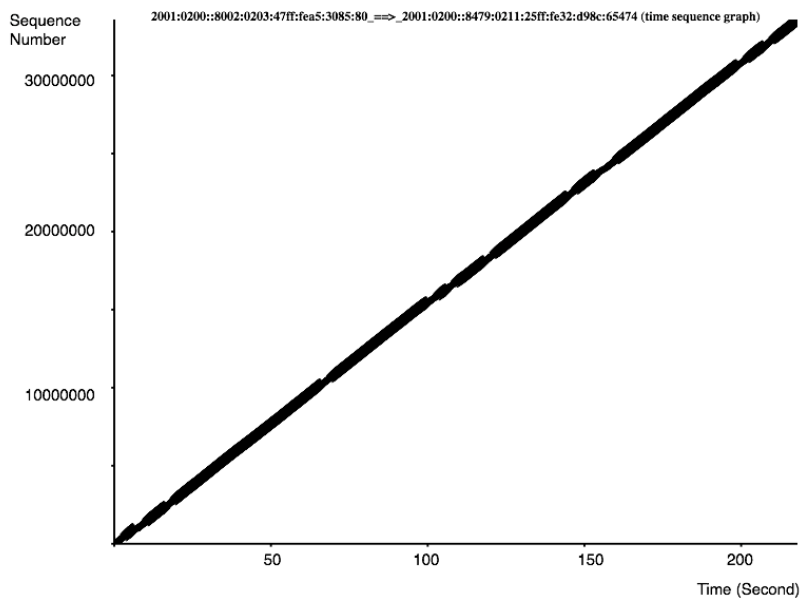

Figure 9. Transition of Sequence Number when the MR was not moving

Figure 10 shows the transition of sequence number when the MR was moving between two T1 links. There were small gaps when the MR moved. Figure 11 is the zoomed image of the first movement in Figure 10. The TCP stream was suspended for about 4 seconds during handover. The same suspension occurred on each handover.

Figure 12 is the result of a TCP stream when the MR was moving between three links (two T1 links and the Satellite link). We can see that the throughput of the TCP stream was lower when the MR was using the Satellite link than the MR was using either of the T1 links. There was two large gaps in 


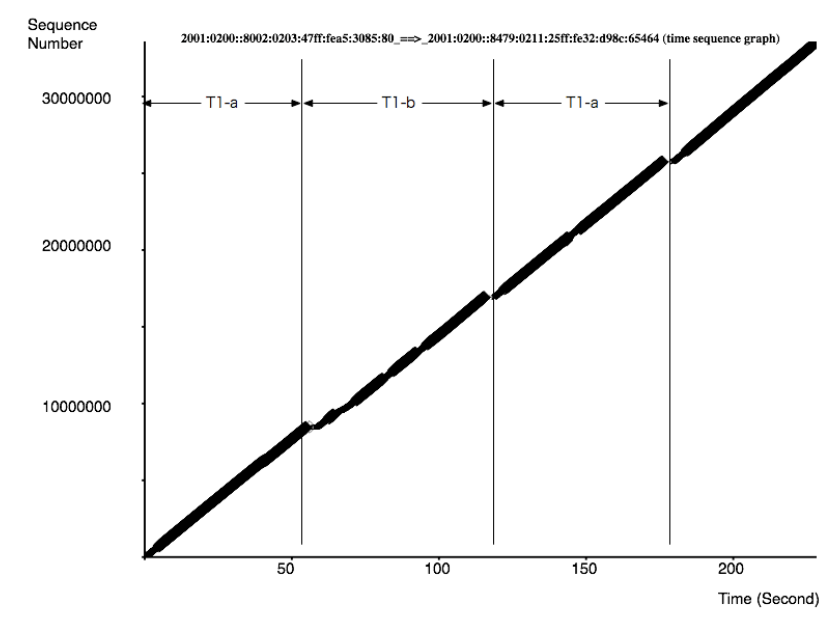

Figure 10. Transition of Sequence Number when the MR was moving between the two T1 links

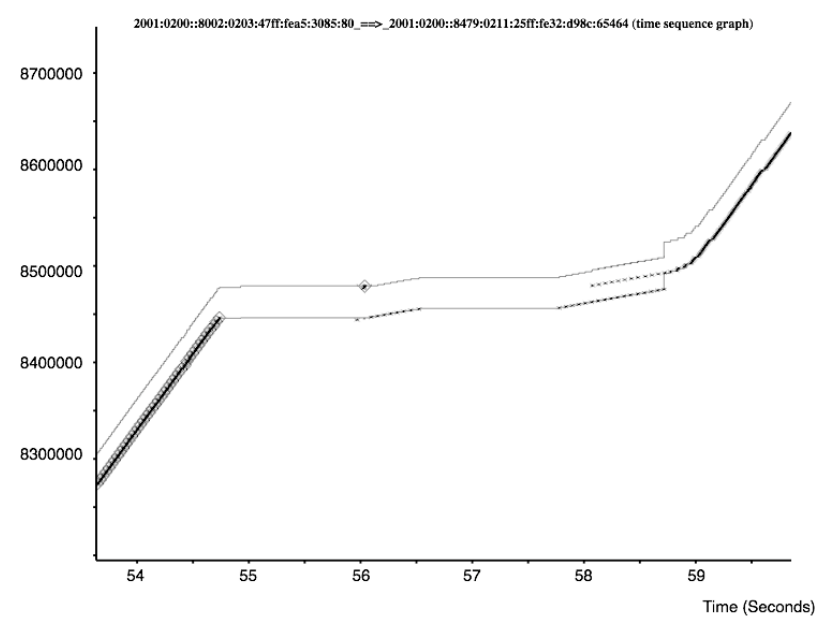

Figure 11. Transition of Sequence Number around the first movement in Figure 10

the graph. The first gap was at the handover time when the MR moved from the Satellite link to the T1 (T1-a) link. The second gap was when the MR moved from the T1-a link to the T1-b link.

\section{COMPARISON AND CONSIDERATION}

We provided a mobile network to a large scale realistic meeting network that had over 250 people in it. The MR moved between two or three different networks without any serious problems and we could provide a moving network to the participants of the meetings. At the first experiment held at the WIDE 2005 autumn meeting, we saw a serious service disruption problem during handover. As the result of ICMPv6 echo request/reply messages shows in TABLE II, there was $31.7 \%$ packet loss from the node inside the meeting network to the node located outside of the meeting network.

In the second experiment held at the WIDE 2006 spring meeting, we introduced the multiple network interfaces at the

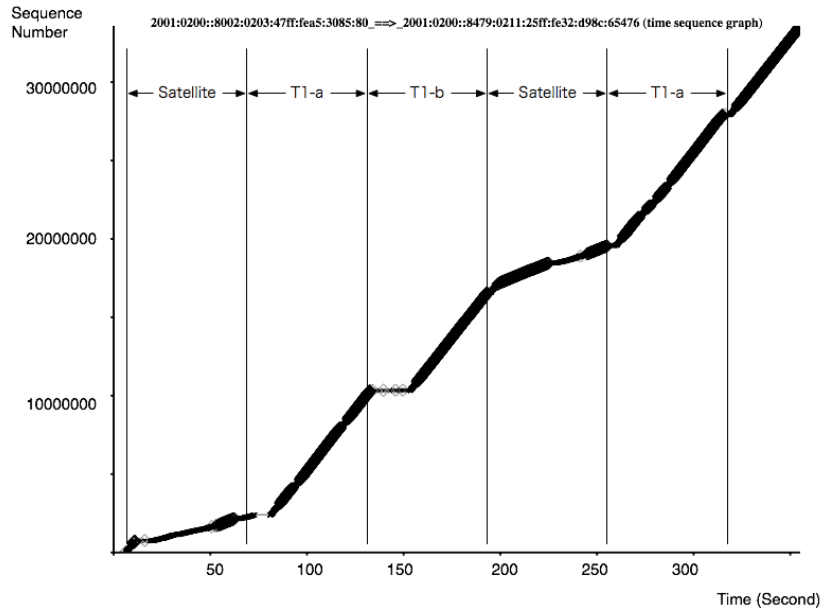

Figure 12. Transition of Sequence Number when the MR was moving between the two T1 and the Satellite links

MR and utilized the Multiple CoAs Registration mechanism. By using the Multiple CoAs mechanism, we could perform the registration procedure for the new CoA that the MR got at the new foreign network before it left the old foreign network. The MR could safely disconnect from the old foreign network after all traffic was routed to the bi-directional tunnel established at the new foreign network. The ICMPv6 echo request/reply result shown as TABLE IV indicates that the mechanism is useful to reduce the packet loss rate. In the second experiment, we only saw $3.2 \%$ to $9.6 \%$ packet loss from the node inside the meeting network. However, considering the fact the MR could use the old bi-directional tunnel before it established a new bi-directional tunnel, the packet loss rate could be smaller than the result.

At the second experiment, we also measured the TCP performance by recording the transition of the sequence number of TCP streams. The total performance of TCP sessions were basically good. We can confirm the result by comparing Figure 9 that depicts the performance of a TCP stream when the MR was not moving and Figure 10 that depicts the performance when the MR was moving between two T1 links. However, when we look at the performance during handover in detail, we can see that the transmission was suspended for about 4 seconds. Assuming that there were 4 seconds suspension for every 60 seconds, that is the movement interval in the second experiment, we will lost 4 ICMPv6 reply packets while sending 60 ICMPv6 echo request packets. The loss rate will be $(4 / 60) \times 100=6.7 \%$. We think the reason of the packet loss shown in TABLE IV is related to the reason of the result of the TCP performance.

At the second meetings, we asked participants of the meetings that how frequently they noticed movement during they were attending the meeting. The result is shown in TABLE V. The result shows that $89.5 \%$ of people did not notice the network movement. 


\begin{tabular}{l|l} 
Frequency & Number of answers \\
\hline \hline Did not notice & 102 \\
\hline Every 10 minutes & 6 \\
\hline Every 5 minutes & 3 \\
\hline Every 2 minutes or more & 3 \\
\hline
\end{tabular}

TABLE V

THE RESULT OF HOW FREQUENTLY PEOPLE FELT THE NETWORK MOVEMENT

\section{CONCLUSION}

When we consider the deployment of a new protocol, there are two important things. One is to implement the specification to find any specification problems that cannot be easily found during designing the specification. The other thing is to use the implemented protocol stack in a real environment to find any operational issues, to have experience of protocol operation and to advertise the new protocol to people. We are trying to deploy the mobility technology especially the network mobility technology based on NEMO BS that is the IETF standard network mobility protocol. The benefit of NEMO BS is that, the nodes inside the moving network served by the mobile router are not required to change its protocol stack to support moving function. The nodes inside the moving network can be normal IPv6 nodes. The typical application of this technology is to provide the Internet to transportation systems such as buses and trains.

We had two experimental operations of mobile network using NEMO BS. We used the WIDE 2005 autumn meeting and the WIDE 2006 spring meeting, those were the actual meeting networks used by the meeting attendees, as the target mobile networks for the experiments. The number of attendees was over 250 people for each meeting, which could be considered as a large moving entity like a train and its passengers. The success of the experiment means the technology can be used for the real transportation systems.

At the first experiment, we used functions that are provided by the base NEMO BS specification only. The short report of the first experiment was also reported as [9]. In the base specification, a mobile router can only use one network interface at one time. That means, when the mobile router moves from one foreign network to the other foreign network, we cannot prevent disconnection of the mobile router. Because of this problem, we had a big packet loss rate $(31.7 \%)$ from the node inside the mobile network provided by the mobile router. In the second experiment, we used an extension of NEMO BS that enables the concurrent use of multiple network interfaces (Multiple CoAs). In the second experiment, our MR was equipped three network interfaces. We defined the local policy of the usage of multiple network interfaces as to connect a new network before leaving from an old network. The result of the packet loss was improved to $3.2 \%$ at the best case. Also, based on the questionary we asked to the meeting attendees showed that most of users did not notice the network movement. We can conclude that the Multiple CoAs mechanism is useful for seamless handover of a mobile network and the mobile network is practically usable as a moving network.
However, as the result of the ICMPv6 packet loss measurement (TABLE IV) and the TCP performance measurement (Figure 11) show, we still have packet loss and TCP transmission suspension problems even if we use the Multiple CoAs mechanism. We will research the reason of these problems and will try to improve the technology to provide smooth moving networks.

\section{REFERENCES}

[1] V. Devarapalli, R. Wakikawa, A. Petrescu, and P. Thubert, "Network Mobility (NEMO) Basic Support Protocol," IETF, Tech. Rep. RFC3963, January 2005.

[2] WIDE project, http://www.wide.ad.jp/.

[3] R. E. Koodli, "Fast Handovers for Mobile IPv6," IETF, Tech. Rep. RFC4068, July 2005.

[4] WIDE project, "SHISA," http://www.mobileip.jp/.

[5] — "KAME Working Group," http://www.kame.net/.

[6] Zebra Project, "GNU Zebra," http://www.zebra.org/.

[7] T. Narten, E. Nordmark, and W. A. Simpson, "Neighbor Discovery for IP Version 6 (IPv6)," IETF, Tech. Rep. RFC2461, December 1998.

[8] R. Wakikawa, T. Ernst, and K. Nagami, "Multiple Careof Addresses Registration," IETF, Tech. Rep. draft-wakikawamobileip-multiplecoa-05, February 2006.

[9] K. Shima, Y. Uo, N. Ogashiwa, and S. Uda, "An operational demonstration of a mobile network with a fairly large number of nodes," in International Symposium on Applications and the Internet Workshops (SAINTW'06). IEEE Computer Society, January 2006, pp. 6-9.

Keiichi Shima graduated the School of Engineering, Kyushu University, Japan, in 1994 and completed the M.S. program at the Graduate School of Information Science, Nara Institute of Science and Technology, Japan in 1996. He is a researcher at Internet Initiative Japan Inc. and engaged in research on the IPv6 unwired mobile networking technologies.

Yojiro Uo, Ph.D, graduated the School of Engineering, Tokyo Institute of Technology, Japan, in 1995 and completed the M.S. program at the Graduate School of Information Science, Japan Advanced Institute of Science and Technology, Japan in 1997. He got the doctor degree of information science from same institution in 2003. He is a researcher at Internet Initiative Japan Inc. and engaged in research on migration of real-space oriented technologies and Internet.

Nobuo Ogashiwa, Ph.D, graduated the Dept. of Systems Engineering, Shibaura Institute of Technology, Japan in 1999 and completed the M.S. program at the Japan Advanced Institute of Science and Technology. He completed the doctoral program in information science at the same institution in 2004. He is now affiliated with Intec NetCore, Inc. He is engaged in research and development related to Internet route control.

Satoshi Uda, Ph.D, graduated the Faculty of Science and Technology, Tokyo University of Science, Japan in 1997 and completed the M.S. program in 1999 at the School of Information Science, Japan Advanced Institute of Science and Technology, where he also completed the doctoral program (in information science) in 2004. $\mathrm{He}$ is a research associate at the Center for Information Science. $\mathrm{He}$ is now engaged in research on enhancement of functionality and operating techniques on the Internet. 